\title{
UNIVERSITY OF TEXAS AT AUSTIN RADIOCARBON DATES XV
}

\author{
S VALASTRO, JR, E MOTT DAVIS, ALEJANDRA G VARELA \\ and SUSAN V LISK
}

\section{Radiocarbon Laboratory, Balcones Research Center}

The University of Texas at Austin

This list begins a program of publishing our large backlog of unpublished dates. The dates listed here are not a specific block of dates but simply those ready for publication. In many cases we have received comments from submitters, to whom we are grateful. Where no comment is included, none was received.

Age calculations are based on the ${ }^{14} \mathrm{C}$ half-life of $5568 \mathrm{yr}$ and modern standard of $95 \%$ oxalic acid, supplemented by tree rings of pre-industrial wood from a $\log$ cut in the 1850 s (Tx-540: R, 1970, v 12, p 249). Deviations reported are based on counting statistics of sample, background and modern, and are $\pm 1 \sigma$, except when sample count approaches either modern or background, $2 \sigma$ limits are reported. Unless noted, ${ }^{12} \mathrm{C} /{ }^{13} \mathrm{C}$ measurements were not made and results are not corrected for ${ }^{13} \mathrm{C}$ fractionation (assumed ratio $=-25 \%$ WRT PDB). Our laboratory uses liquid scintillation counting of benzene, with $\mathrm{Li}_{2} \mathrm{C}_{2}$ and vanadium-activated catalyst in preparation; chemical yields range between $95 \%$ and $99 \%$. Three counters are employed: a Packard Tri-Carb Model 3002 and 2 Beckman LS230 spectrometers obtained through a grant from the National Science Foundation.

\section{ACKNOWLEDGMENTS}

We acknowledge with gratitude the assistance of Carol Vernon in preparing the date list, and the administrative support and assistance of Dean Robert E Boyer of the College of Natural Sciences, and the staff of the Texas Memorial Museum.

\section{GEOLOGIC SAMPLES}

\section{United States}

\section{Tx-4259. Natural Lake, Oklahoma}

$1040 \pm 70$

Mud from 53 to $58 \mathrm{~cm}$ in core sample III, from base of Natural Lake, $2.5 \mathrm{~km}$ E of Sardis, N of Jackfork Creek, Pushmataha Co $\left(34^{\circ} 2^{\prime} \mathrm{N}, 95^{\circ} 1^{\prime}\right.$ W). Paleobot sample, coll 1977 by D G Wyckoff, P J Mehringer, L E Albert; subm 1981 by Albert, Oklahoma Archeol Survey, Univ Oklahoma, Norman. Comment (LEA): date regarded as accurate and useful in reconstruction of local vegetational and environmental settings (Albert, 1981).

\section{Nevada}

\section{Helms Pit series}

Samples from Helms gravel pit, city of Sparks $\left(39^{\circ} 32^{\prime} 10^{\prime \prime} \mathrm{N}, 119^{\circ} 43^{\prime}\right.$ $\left.46^{\prime \prime} \mathrm{W}\right)$, to provide new control on Late Pleistocene and Holocene glacial 
outwash sequence from Sierra Nevada. Coll and subm 1982 by J W Bell, Nevada Bur Mines \& Geol, Univ Nevada, Reno.

Tx-4699. Helms V-2

$23,700 \pm 580$

Log from silt lens $13.7 \mathrm{~m}$ below surface in NW corner of pit.

Tx-4700. Helms V-6

$9150 \pm 120$ Tx-4699.

Twigs from sand lens $7.6 \mathrm{~m}$ below surface in SE corner of pit, overlying

Tx-4701. Helms V-7

$6860 \pm 110$

Peaty material from sandy silt lens ca $6 \mathrm{~m}$ below surface in $\mathrm{SE}$ corner, overlying Tx-470).

Tx-4702. Helms V-8

$7990 \pm \mathbf{5 3 0}$ 4701 .

Peat from peat lens ca $4.6 \mathrm{~m}$ below surface in SE corner, overlying Tx-

Tx-4703. Helms V-9

Modern 47() 2 .

Peat from peat layer ca $2 \mathrm{~m}$ below surface in SE corner, overlying Tx-

General Comment (JWB): pre-modern dates are from sand and gravel deposit of Truckee $\mathrm{R}$, interpreted here as Tioga outwash. Tx-4701 and -4702 dates appear reversed from strat position.

\section{Fallon Trench \# 1 series}

Detrital and disseminated charcoal samples from backhoe trench excavated linear dune-covered ridge, $20 \mathrm{~km} \mathrm{~S}$ of Fallon $\left(39^{\circ} 18^{\prime} 30^{\prime \prime} \mathrm{N}, 118^{\circ} 49^{\prime}\right.$ $20^{\prime \prime} \mathrm{W}$ ). Coll and subm 1980 by J W Bell.

\section{Tx-4079. Fallon 1}

$1550 \pm 140$

From base of Holocene soil (camborthid) developed on Holocene-age Fallon fm in unfaulted sediments overlying fault, and interpreted to overly Turupah Flat Ash; should give min ages for faulting and for deposition of Turupah Flat Ash.

\section{Tx-4080. Fallon 2}

$1680 \pm 110$

From faulted lacustrine and/or colian sediments of Fallon $\mathrm{fm} 0.6 \mathrm{~m}$ below Turupah Flat Ash; should determine recency of faulting and max age for Turupah Flat Ash.

General Comment (JWB): dates bracket age of Turupah Flat Ash, a widespread tephra in central and W Nevada.

\section{Tx-4213. Carson Dam Trench 1}

$11,660 \pm 220$

Calcareous dendritic tufa from trench across fault on Lake Lahontan shoreline, $4140 \mathrm{amsl}, 3.9 \mathrm{~km} \mathrm{SW}$ of Carson Diversion Dam (39 $27^{\prime} 48^{\prime \prime} \mathrm{N}$, $119^{\circ}\left(00^{\prime} 48^{\prime \prime} \mathrm{W}\right)$. Should give max age of movement on major fault zone. Coll and subm 1980 by J W Bell. Comment (JWB): sample dates dendritic 
member of Sehoo fm; in good agreement with other tufa dates from this unit (Broccker \& Kaufman, 1965).

Tx-4960. Sha Neva LV-1

$10,440 \pm 490$

Mammoth rib (Mammuthus sp) from Sha Neva gravel pit, $4.8 \mathrm{~km} \mathrm{NE}$ of Reno-Stcad Airport, Washoe Co, 4980 amsl (39 40 46 $46^{\prime \prime} \mathrm{N}, 119^{\circ} 48^{\prime} 36^{\prime \prime}$ W). Should date last major stand of Late Pleistocene Lake Lemmon. Coll 1983 by (G Szecsody and I) Bryan, and subm 1983 by J W Bell. Comment (JWB): date agrees well with ages obtained on last high stands of Lake Lahontan, which was probably contemporaneous with Lake Lemmon.

\section{Australia}

\section{UDP-1 series, Northern Territory}

Samples from Holocene overbank deposits, Uranium Development Proj Loc 1, 2.6km SE of UDP Falls, N bank South Alligator R $\left(13^{\circ} 27^{\prime} \mathrm{S}\right.$, $132^{\circ} 26^{\prime} \mathrm{E}$ ). Coll and subm 1980 by V R Baker, Dept Geol Sci, Univ Texas, Austin.

\section{Tx-4207. UDP $1 / \mathrm{W}$}

$$
\begin{aligned}
& \delta^{14} \mathrm{C}=+353.62 \pm 7.9 \% 0 \\
& \text { Ultramodern } \\
& \delta^{13} \mathrm{C}=-25.9 \pm 0.2 \%
\end{aligned}
$$

Wood, $1 \mathrm{~m}$ below land surface, above contact with gray layer.

Tx-4211. UDP 1/C

$280 \pm 100$

Charcoal, $1 \mathrm{~m}$ below surface at top of gray layer.

\section{Tx-4212. UDP 1/G}

Charcoal, $1.3 \mathrm{~m}$ below surface within gray layer.

General Comment (VRB): samples give rates of overbank sedimentation (Baker, Pickup \& Polach, 1983).

\section{Finke River series, Northern Territory}

Samples from Holocene slackwater sediments, from E bank Finke R, S of Hermannsburg. Coll 1980 by G Pickup; subm 1980 by V R Baker.

Tx-4208. Finke 2/2L5

$$
\delta^{14} \mathrm{C}=+\begin{array}{r}
336.01 \pm 29.5 \% 0 \\
\text { Ultramodern }
\end{array}
$$

Charcoal, $2 \mathrm{~m}$ below surface in Layer $5,12 \mathrm{~km} \mathrm{~S}$ of Hermannsburg $\left(24^{\circ} 03^{\prime} \mathrm{S}, 132^{\circ} 46.5^{\prime} \mathrm{E}\right)$. Comment (VRB): date agrees with dates ANU2400), -2402, and -2403 (Baker, Pickup \& Polach, 1983).

\section{Tx-4209. Finke 4/1L1R}

$\delta^{14} \mathrm{C}=+48.01 \pm 20.4 \%$ Ultramodern

Woody material, $20 \mathrm{~cm}$ below surface, $4 \mathrm{~km} \mathrm{~S}$ of Hermannsburg $\left(23^{\circ}\right.$ $\left.59^{\prime} \mathrm{S}, 132^{\circ} 46.5^{\prime} \mathrm{E}\right)$. Comment (VRB): sample coll and dated to correlate with Tx-4208; correlation is confirmed. 
General Comment (VRB): samples confirm recent phase of desert floods on Finke R.

\section{OCEANOGRAPHIC SAMPLES}

\section{Orca Basin series, Louisiana}

Samples from Orca basin, emponded brine basin on lower continental slope off W Louisiana. Coll 1978 by PL Parker and JL Worzel, and subm 1978 by E W Behrens, Univ Texas Marine Sci Inst Geophysics Lab, Galveston. $\mathrm{Cm}$ figures are depths in core of center of sample.

Tx-3174. IG27-04-155

$11,920 \pm 150$

Core $4\left(27^{\circ} 00.3^{\prime} \mathrm{N}, 91^{\circ} 23.4^{\prime} \mathrm{W}\right), 155 \mathrm{~cm}$.

Tx-3175. IG27-02-485

$7910 \pm 170$ mud.

Core $2\left(26^{\circ} 55.6^{\prime} \mathrm{N}, 91^{\circ} 19.5^{\prime} \mathrm{W}\right), 485 \mathrm{~cm}$. Gas-rich laminated black

Tx-3176. IG27-02-570

$10,320 \pm 200$

Core 2, same loc as Tx-3175; $570 \mathrm{~cm}$. Gas-poor massive gray mud.

Tx-3177. IG27-02-745

$17,380 \pm 410$

Core 2, same loc as $T \mathrm{x}-3175 ; 745 \mathrm{~cm}$.

General Comment (EWB): Tx-3174 gives sedimentation rate for normal continental slope next to basin. Tx-3175 and -3176 give rate for basin. All three are estimated to be basal Holocene. Tx-3177 is uppermost Pleistocene at major peak in manganese concentration in core, interpreted to represent major change in sedimentation rate, possibly when basin changed from normal slope environment to emponded brine basin.

\section{Tx-3857. Sugar Bay D1-D2, Virgin Islands}

$3600 \pm 140$

Shells (gastropod, Halimeda thalli) from $230 \mathrm{~cm}$ sub-bottom depth in Core D, mid-channel core $300 \mathrm{~m}$ from head of Sugar Bay on Salt R estuary, St Croix ( $\left.17^{\circ} 48^{\prime} \mathrm{N}, 64^{\circ} 44^{\prime} \mathrm{W}\right)$. Coll 1978 and subm 1980 by R W Arnseth, Dept Geol Sci, Northwestern Univ, Evanston, Illinois. Comment (RWA): sample dates sedimentation rate for documentation of diagenetic change rates. Core penetrated marine grass bank deposit; linear sedimentation rate calculated to be $64 \mathrm{~cm} / 1000 \mathrm{yr}$.

Tx-2205. Harrington Sound, Bermuda

$850 \pm 120$

Argopecten gibbus scallop from cores 75-9 and 75-12, 45 to $55 \mathrm{~cm}$ below sediment-water interface, ca $24 \mathrm{~m}$ below surface of Harrington Sound, $0.4 \mathrm{~m} \mathrm{NW}$ of Devil's Hole village ( $\left.32^{\circ} 19^{\prime} 20^{\prime \prime} \mathrm{N}, 64^{\circ} 43^{\prime} 00^{\prime \prime} \mathrm{W}\right)$. Subm to examine faunal transition from Argopecten to Gouldia. Coll 1975 and subm 1975 by G R Clark, Dept Geol Sci, State Univ Coll, Geneseo, New York.

\section{Continental Shelf series, Brazil}

Samples from various loci, subm to establish preliminary reconstruction of Late Quaternary history of $\mathrm{N}$ and $\mathrm{W}$ sectors of Brazilian continental 
shelf. Coll 1971 to 1974 and subm 1975 by H A F Chaves, Petrobras, Petróleo Brasileiro S A, New York City. Depth of water at site given after type of material.

Tx-2288. GEOMAR III-E 2533-ID

$7960 \pm 90$

Oyster shell, $21 \mathrm{~m}$, continental shelf off Salinópolis $\left(0^{\circ} 29.0^{\prime} \mathrm{S}, 47^{\circ}\right.$ 24.0' W); surface sample.

Tx-2289. GEOMAR III-E 2467-1B

$2290 \pm 60$

Shell (Tivela fulminata), $42 \mathrm{~m}$, continental shelf off Salinópolis $\left(0^{\circ} 52.5^{\prime}\right.$ $\left.\mathrm{N}, 47^{\circ} 16.0^{\prime} \mathrm{W}\right)$; surface sample.

Tx-2290. GEOMAR III-E 2496-2B

$$
\delta^{14} \mathrm{C}=+18.20 \pm 3.5 \%
$$

Ultramodern

Oyster shell, $47 \mathrm{~m}$, continental shelf off Amapá $\left(3^{\circ} 32.0^{\prime} \mathrm{N}, 50^{\circ} 24.0^{\prime}\right.$ W); surface sample.

Tx-2291. GEOMAR III-G 193-2D

$7940 \pm 110$

Shell (Plicatula gibbosa), 73m, continental shelf off Amapá $\left(3^{\circ} 37.0^{\prime} \mathrm{N}\right.$, $\left.50^{\circ} 1.0^{\prime} \mathrm{W}\right)$; surface sample.

Tx-2292. GEOMAR III-G 215-2D

$10,790 \pm 110$

Vermetidae and carbonate matrix, $122 \mathrm{~m}$, shelf break off Amapá (4 $\left.6.0^{\prime} \mathrm{N}, 49^{\circ} 36.0^{\prime} \mathrm{W}\right)$; surface sample.

\section{Tx-2293. REMAC-AR-T 4294/1}

$18,750 \pm 280$

Shell hash, $73 \mathrm{~m}$, inner shelf off coastal plain between Niteroi and Cabo Frio, off Rio de Janciro $\left(23^{\circ} 8.0^{\prime} \mathrm{S}, 42^{\circ} 44.1^{\prime} \mathrm{W}\right) ; 45 \mathrm{~cm}$ from top of $64 \mathrm{~cm}$ piston core.

Tx-2294. REMAC-AR-T 4343/3

$6470 \pm 70$

Shell, $19 \mathrm{~m}$, Albardão Bank, continental shelf off Rio Grande do Sul $\left(32^{\circ} 59.5^{\prime} \mathrm{S}, 59^{\circ} 23.2^{\prime} \mathrm{W}\right) ; 55 \mathrm{~cm}$ from top of $151 \mathrm{~cm}$ piston core. Upper $40 \mathrm{~cm}$ of core was inner transgressive sand sheet.

Tx-2295. REMAC-AR-T 4160/5

$10,620 \pm 300$

Shell, 66m, Abrolhos Bank, continental shelf off Espirito Santo (18 $45.8^{\prime} \mathrm{S}, 38^{\circ} 33.6^{\prime} \mathrm{W}$ ); $95 \mathrm{~cm}$ from top of $141 \mathrm{~cm}$ piston core. Terrigenous mud facies just below contact with upper calcareous mud facies.

Tx-2296. REMAC-AR-T 4324/6

$$
17,330 \pm 480
$$

Shell covered by calcareous algae, $132 \mathrm{~m}$, mid-outer shelf off Rio Grande do Sul $\left(31^{\circ} 10.5^{\prime} \mathrm{S}, 49^{\circ} 55.3^{\prime} \mathrm{W}\right) ; 180$ to $190 \mathrm{~cm}$ from top of $237 \mathrm{~cm}$ piston core. From coarse biogenic layer. Algae covering shell indicates climatic change.

Tx-2297. REMAC-AR-T 4304/7

$12,550 \pm 140$

Shell, oyster, and shell hash, 105m, mid-shelf off São Paulo $\left(24^{\circ} 55.0^{\prime}\right.$ $\left.\mathrm{S}, 45^{\circ} 13.5^{\prime} \mathrm{W}\right) ; 150 \mathrm{~cm}$ from top of $167 \mathrm{~cm}$ piston core. Lower part of core 
has rich biogenic assemblage; grades upward to pure sand without biogenic components.

Tx-2298. REMAC-AR-T 4333/11a

$14,700 \pm 170$

Shell hash, $55 \mathrm{~m}$, outer continental shelf, S Rio Grande do Sul $\left(33^{\circ}\right.$ $\left.44.7^{\prime} \mathrm{S}, 51^{\circ} 34.8^{\prime} \mathrm{W}\right) ; 360 \mathrm{~cm}$ from top of $388 \mathrm{~cm}$ piston core; sandy mud facies with evidence of reworking during lowered sea level stand.

Tx-2300. REMAC-AR-T 4319/4a

$19,900 \pm 330$

Shell hash, $115 \mathrm{~m}$, mid-shelf off Santa Catarina $\left(28^{\circ} 40.05^{\prime} \mathrm{S}, 48^{\circ} 9.0^{\prime}\right.$ W); $150 \mathrm{~cm}$ from top of $200 \mathrm{~cm}$ piston core. Bottom of transgressive Holocene sequence.

Tx-2301. REMAC-AR-T 4322/5a

$42,100 \pm 3900$

Coral, $528 \mathrm{~m}$, continental slope off Rio Grande do Sul ( $30^{\circ} 51.0^{\prime} \mathrm{S}, 49^{\circ}$ $\left.3.2^{\prime} \mathrm{S}\right) ; 190 \mathrm{~cm}$ from top of $200 \mathrm{~cm}$ piston core. Coral layer was covered by slope mud.

Tx-2302. GIII-152-ID

$7470 \pm 70$

Carbonate rock, $125 \mathrm{~m}$, shelf break off Salinópolis $\left(2^{\circ} 29.5^{\prime} \mathrm{N}, 47^{\circ}\right.$ $\left.36.5^{\prime} \mathrm{W}\right)$; dredged surface sample. Relict sediment; oldest-looking carbonate rocks found at shelf break.

Tx-2303. GVI-325

$21,100 \pm 430$

Shell hash, $25 \mathrm{~m}$, shell bank, inner continental shelf off Rio Grande, Rio (srande do Sul (32 $\left.13^{\prime} \mathrm{S}, 51^{\circ} 46^{\prime} \mathrm{W}\right)$; surface sample, side of shoal. Probable ancient strandline of late Holocene age.

\section{Tx-2304. GVI-362}

$17,420 \pm 270$

Shell hash, $135 \mathrm{~m}$, shell debris facies, outer continental shelf off Rio Grande do Sul ( $\left.31^{\circ} 6^{\prime} \mathrm{S}, 49^{\circ} 46^{\prime} \mathrm{W}\right)$; surface sample. May be oldest and deepest exposed strandline deposits in this part of coastal plain.

Tx-2305. REMAC-AR-4335

$23,050 \pm 550$

Shell hash, $28 \mathrm{~m}$, Albaradão Bank, inner shelf off Rio Grande do Sul $\left(33^{\circ} 27.4^{\prime} \mathrm{S}, 52^{\circ} 27.0^{\prime} \mathrm{W}\right)$; surface sample. Possible marker of ancient shoreline.

Tx-2306. REMAC-AR-4277

$7450 \pm 210$

Oyster and other shell, $28 \mathrm{~m}$, continental shelf off Campos, $\mathrm{S}$ of Paraiba do Sul delta $\left(22^{\circ} 12.0^{\prime} \mathrm{S}, 41^{\circ} 2.7^{\prime} \mathrm{W}\right)$; surface sample. Relict coarse sand facies reflecting competence of sediment source not found today.

Tx-2307. WHOI-AR-3133

$13,780 \pm 170$

Shell hash, $136 \mathrm{~m}$, outer continental shelf off Santa Catarina $\left(27^{\circ} 28^{\prime} \mathrm{S}\right.$, $47^{\circ} 34^{\prime} \mathrm{W}$ ); surface sample. Thought to be relict near-shore deposit; should give max age of shoreline. 
Tx-2407. REMAC-AR-T 4322/2

Coral, $528 \mathrm{~m}$, continental slope off Rio (srande do Sul $\left(30^{\circ} 51.05^{\prime} \mathrm{S}\right.$, $\left.49^{\circ} 3.2^{\prime} \mathrm{W}\right) ; 25 \mathrm{~cm}$ from top of $200 \mathrm{~cm}$ piston core. Coral layer was covered by slope mud.

\section{G-2 series, Persian Gulf}

Marine shells (mixture of spp) from boring G-2 (25 $\left.36^{\prime} \mathrm{N}, 54^{\circ} 28^{\prime} \mathrm{E}\right)$, water depth $38 \mathrm{~m}$. Coll and subm 1977 by H Chafetz, Geol Dept, Univ Houston, Houston, Texas.

\section{Tx-2824. G-2, S-21}

From ca $71.3 \mathrm{~m}$ below msl.

Tx-2825. G-2, S-24

From ca $75.6 \mathrm{~m}$ below msl. $>\mathbf{4 0 , 0 0 0}$

$$
\delta^{13} C=2.7 \pm 0.2 \%
$$

$>40,000$

$$
\delta^{13} C=2.1 \pm 0.2^{\%} \%
$$

ARCHAFOLOGIC SAMPLES

United Slates

Texas

Texas dates are arranged geographically, $\mathrm{W}$ to E: trans-Pecos, southcentral and south, coast, northeast.

\section{Gobernadora site series}

Charred wood from Gobernadora site, $41 \mathrm{EP} 321$, in right of way of Northeast Expressway, NE El Paso $\left(31^{\circ} 56^{\prime} \mathrm{N}, 106^{\circ} 25^{\prime} \mathrm{W}\right)$. Late Mesilla phase, Jornada Branch, Mogollon. Coll 1981-82 and subm 1982 by J W Clark, Texas Dept Hwys \& Public Transportation, Austin.

Tx-4713. 41EP321/19

$750 \pm 130$

Probable roof-beam fragment, fill of Pithouse \#2; N60/E22, Lcvel 7.

Tx-4714. 41EP321/59

$1120 \pm 40$ Level 7.

Probable roof-bcam fragment, floor of Pithouse \#1; N58/W2,

Tx-4715. 41EP321/163

$1070 \pm 180$

Charcoal from hearth; N30/W40; Level 2.

Tx-4716. 41EP321/332

$790 \pm 60$

Charcoal from hearth; N68/E8, Level 5.

Tx-4717. 41EP321/345

$1440 \pm 60$

Charcoal from hearth; N68/E6, Level 1. 
Tx-4718. 41EP321/350

Charcoal from hearth in floor of Pithouse \#3; N66/E8.

Tx-4719. 41EP321/464

$\mathbf{9 7 0} \pm \mathbf{8 0}$

Probable roof-beam fragment, floor fill of Pithouse \#2; N60/E20, Level 7.

Tx-4720. 41EP321/423

$1110 \pm 60$

Charcoal from hearth; N32/E12, Level 1.

\section{Ojasen site series}

Charcoal from Ojasen site, 41EP289, in right of way of Northeast Expressway, NE El Paso $\left(31^{\circ} 56^{\prime} \mathrm{N}, 106^{\circ} 25^{\prime} \mathrm{W}\right)$. Late Mesilla phase, Jornada Branch, Mogollon. Coll 1981 and subm 1983 by J W Clark.

Tx-4863. 41EP289/283

$1230 \pm 70$

From hearth outside pithouse, S32/E8; El Paso Brown, El Paso Polychrome and Mimbres Black-on-White ceramics assoc.

Tx-4864. 41EP289/295

$1490 \pm 120$

From pithouse floor, S32/E10; El Paso Brown, El Paso Polychrome and Mimbres ceramics assoc.

Tx-4865. 41EP289/176

$1260 \pm 70$

From trash pit assoc with pithouses, S34/E12, base of Level 2; El Paso Brown and El Paso Polychrome ceramics assoc.

\section{Tx-4866. 41EP289/31}

$1120 \pm 130$

From probable base of pithouse floor, S20/W8, Level 1; El Paso Brown and El Paso Polychrome ceramics assoc.

\section{Tx-2806. 41CU97/F5}

$710 \pm 50$

Charcoal from within wall of ring midden, Feature 5 , in multi-midden site, 41 CU97 (Katz, 1978), 200m S of Texas-New Mexico state line beside Dog Canyon arroyo in Guadalupe Mts Natl Park, Culberson Co $\left(31^{\circ} 60^{\prime} \mathrm{N}\right.$, $104^{\circ} 50^{\prime} \mathrm{W}$ ). Assoc materials include Perdiz and Livermore points, Jornada Brown and El Paso Polychrome sherds, all of which suggest Late Prehistoric, Jornada Mogollon affiliation. Coll 1976 and subm 1977 by S R Katz, Center Archaeol Research, Univ Texas, San Antonio. Comment (SRK): date verifies assocs (Katz, 1978, p 87).

\section{BS609 series}

Charcoal from Site 41 BS609, both sides of U S Hwy $385,0.8 \mathrm{~km} \mathrm{~S}$ of entrance to Big Bend Natl Park, Brewster Co $\left(29^{\circ} 40^{\prime} \mathrm{N}, 103^{\circ} 10^{\prime} \mathrm{W}\right)$. Coll 1977 and subm 1977 by B J Baskin, Texas Archeol Research Lab, Univ Texas, Austin. 
Tx-2869. 41BS609/3

Hearth, W half Unit N504/E492, Level 3, 98.85m elev. No diagnostic artifacts assoc.

Tx-2870. 41BS609/7

$1060 \pm 50$

Bottom of lowest occupation zone in test pit, Unit N491/E496, Level $4,99.14$ to $99.09 \mathrm{~m}$ elev. No diagnostic artifacts assoc.

General Comment (BJB): dates place occupation of site either from Late Archaic through Late Prehistoric, or within purely Late Prehistoric period (Baskin, 1978, p 34).

\section{Upper Four Mile Draw site series}

Charcoal from Hearth 1, Area 4, at 41 PC395, Upper Four Mile Draw site, $19.5 \mathrm{~km} \mathrm{~W}$ of Sheffield, Pecos Co $\left(30^{\circ} 45^{\prime} 10^{\prime \prime} \mathrm{N}, 101^{\circ} 58^{\prime} 5^{\prime \prime} \mathrm{W}\right)$. Late Prehistoric affiliation. Coll and subm 1980 by G M Canon, 850 Gaylord, Denver, Colorado.

Tx-4154. 41PC395/4

$460 \pm 90$

From $8 \mathrm{~cm}$ below ground surface.

Tx-4155. 41PC395/12

$1390+110$

From $16.5 \mathrm{~cm}$ below ground surface.

General Comment (GMC): Tx-4155 too old for Late Prehistoric; may indicate multiple use of hearth.

\section{Tx-2805. 41BX305/c-3/40-50}

$1230 \pm 50$

Charcoal from Site $41 \mathrm{BX} 305$ (Katz, 1977), in John James Park, W bank Salado Creek in NE San Antonio, Bexar Co $\left(29^{\circ} 29^{\prime} 35^{\prime \prime} \mathrm{N}, 98^{\circ} 25^{\prime} 10^{\prime \prime}\right.$ W). From Sq C-3, 40 to $50 \mathrm{~cm}$ below surface, in burned rock concentration with Middle Archaic (Montell, Castroville, Bulverde) points. Coll and subm 1977 by S R Katz. Comment (SRK): dates small activity area; consistent with other dates for $\mathrm{S}$ central Texas Archaic.

Tx-2815. 41BX36/5-2

$300 \pm 120$

Animal bone from Unit 5,10 to $20 \mathrm{~cm}$ below surface, Site $41 \mathrm{BX} 36$ (Gerstle, Kelly \& Assad, 1978), Late Prehistoric site (Perdiz arrow point assoc) on large terrace overlooking Salado Creck near Camp Bullis headquarters in San Antonio (29 38 $8^{\prime} 05^{\prime \prime} \mathrm{N}, 98^{\circ} 34^{\prime} 10^{\prime \prime} \mathrm{W}$ ). Coll 1977 by C Assad and subm 1977 by T R Hester, Center Archaeol Research, Univ Texas, San Antonio. Comment (TCK): sample subm for comparison with charcoal date RL-817 (420 \pm 120; Gerstle, Kelly \& Assad, 1978, p 253); dates are comparable.

\section{Panther Springs Creek site series}

Wood charcoal from Panther Springs Creek site, 41 BX228, E bank Panther Springs Creek, N of San Antonio, Bexar Co $\left(29^{\circ} 33^{\prime} 8^{\prime \prime} \mathrm{N}, 98^{\circ} 30^{\prime}\right.$ $10^{\prime \prime} \mathrm{W}$ ). Coll 1977 and 1979 by T R Hester and S L Black; subm by T R Hester. 
Tx-2810. 41BX228/A-2

$480 \pm 140$

Unit N $100 /$ W99, SE quad, 10 to $20 \mathrm{~cm}$ below surface. Assoc with Late Archaic and Late Prehistoric projectile points.

Tx-2811. 41BX228/A-3

$940 \pm 180$

Same loc as Tx-2810, 20 to $30 \mathrm{~cm}$ below surface. No assoc artifacts.

Tx-2812. 41BX228/C-3

$1110 \pm 110$

Unit N108/W104, Level $3(20$ to $30 \mathrm{~cm}$ below surface). Assoc with Late Archaic Frio point.

Tx-3852. 41BX228/19

$2660 \pm 60$

$\operatorname{Tr} 4, \mathrm{~N}$ wall profile in vertical center of midden, 16 to $20 \mathrm{~cm}$ below surface, $98.82 \mathrm{~m}$ elev. No diagnostic assocs, but below Late Archaic, above preArchaic; thus, Early to Middle Archaic.

Tx-3853. 41BX228/22

$4300 \pm 130$

Area C, E.1005.8/N1024.9, 58cm below surface, $98.92 \mathrm{~m}$ elev. From lowest occupation level in $\mathrm{N}$ area of site; Early and pre-Archaic points from adjacent units on same level.

Tx-3854. 41BX228/1

$1030 \pm 70$

Area A, Unit E1017/N 1020 vicinity, 11 to $16 \mathrm{~cm}$ below surface, 100.05 to $100.0 \mathrm{~m}$ elev. From Feature 1 (burned rock accumulation) fill; Late Prehistoric points and pottery assoc.

Tx-3855. 41BX228/20

$1010 \pm 150$

Area B, E996.88/N1011.45, 25cm below surface, $99.34 \mathrm{~m}$ elev. From fill of Feature 3 (baked clay mass); I ate Archaic Ensor point assoc.

Tx-3856. 41BX228/17

$980 \pm 60$

Area A, E1015.83/N1018.6, 31 cm below surface, $98.81 \mathrm{~m}$ clev. From Feature 5 (baked clay mass) fill; Middle Archaic Pedernales point assoc.

Tx-3911. 41BX228/10

$240 \pm 60$

Area B, E998.61/N1010.2, 20cm below surface, 99.5m elev. Assoc with Feature 2 (burned limestone cluster with metate). Transitional Archaic (Twin Sisters phase) points found in adjacent units at same level.

Tx-3912. 41BX228/31

$4720 \pm 170$

Area I, Unit E994/N969, Level 9 (98.6 to $98.5 \mathrm{~m}$ elev), $84 \mathrm{~cm}$ below surface. Lower transition zone with pre-Archaic (San Geronimo phase) tools; earliest cultural component found at site.

Tx-2774. 41 KL30/JLR-3

$1960 \pm 100$

Charcoal from base of hearth, $60 \mathrm{~cm}$ below surface, Scarborough site, $41 \mathrm{KL} .30$, $9 \mathrm{~km}$ E of Ricardo, Klcberg Co, S Texas $\left(27^{\circ} 25^{\prime} 40^{\prime \prime} \mathrm{N}, 97^{\circ} 45^{\prime} 40^{\prime \prime}\right.$ W). Late Prehistoric, Aransas focus. Coll and subm 1977 by J I. Russell, Geog \& ( reol Dept, Texas A and I Univ, Kingsville. 


\section{GD21 series}

Charcoal from 41GD21, Archaic through Late Prehistoric sitc, midway between Victoria and Goliad, SW side Sulphur Creek, $1.2 \mathrm{~km}$ upstream from Perdido Creek, Goliad Co $\left(28^{\circ} 43^{\prime} 30^{\prime \prime} \mathrm{N}, 97^{\circ} 12^{\prime} 15^{\prime \prime} \mathrm{W}\right)$. Coll and subm 1978 by D E Fox, Center Archaeol Research, Univ Texas, San Antonio. Levels are $20 \mathrm{~cm}$ excavation levels, numbered from surface down.

$$
\begin{array}{llr}
\text { Tx-2923. 41GD21/17B } & \delta^{14} \mathrm{C}=+\begin{array}{r}
\mathbf{3 8 0 . 5 7} \pm \mathbf{1 2 . 4 \% 0} \\
\text { Ultramodern }
\end{array} \\
\text { Unit N107/E210, Level 6, lower portion Zone B. }
\end{array}
$$

Tx-2924. 41GD21/17A

$4260 \pm 250$

Unit N207/E210, Level 6, lower portion Zone B.

Tx-2925. 41GD21/28

$2660 \pm 370$

Unit N208/E210, Level 2, lower portion Zone A.

Tx-2926. 41GD21/15

$4550 \pm 190$

Unit N208/E210, Level 5, lower portion Zone B.

General Comment (DEF): Tx-2924 and -2926 support Early to Middle Archaic affiliation of material within Zone B. Tx-2925 confirms Late Archaic age of Zone A, assoc with "Morhiss Complex" materials (Fox, $1979, \mathrm{p} 62)$.

\section{Palmetto Bend Reservoir}

Samples from basin of Palmetto Bend Rescrvoir on Navidad R in Jackson Co, central coast of Texas. Coll 1976, 1977, and subm 1977 by W B Fawcett, Jr, Texas Archeol Survey, Univ Texas, Austin.

\section{Chytka site series}

Burned bone (except Tx-2752) from Chytka site, 41JK66, Late Archaic to Late Prehistoric site on N side Mustang Creek, $1.6 \mathrm{~km}$ SE of Ganado, in Palmetto Bend Reservoir basin (29 $\left.2^{\prime} \mathrm{N}, 96^{\circ} 29^{\prime} \mathrm{W}\right)$. Residue from screening.

Tx-2752. 41JK66/1

$2210 \pm 70$

Wood/charcoal, Zone 4. Probable Late Archaic period.

Tx-2754. 41JK66/10

$1470 \pm 170$

N16-17/E14-17, Zones 1 and 2.

Tx-2755. 41JK66/11

$770 \pm 100$

N14-15/E14-17, Zones 1 and 2.

Tx-2756. 41JK66/13

$1320 \pm 90$

N14-15/E16, Zone 3A. Austin phase, Late Prehistoric period. 
Tx-2757. 41JK66/14

$510 \pm 100$

N12-13/E14-15, Zone 3A. Austin phase, Late Prehistoric period.

Tx-2758. 41JK66/15

$1520 \pm 100$

N14-15/E12-13, Zone 3. Austin phase, Late Prehistoric period.

Tx-2759. 41JK66/18

$790 \pm 200$

N16-17/E16-17, Zone 3A. Austin phase, Late Prehistoric period.

Tx-2760. 41JK66/19

$1620 \pm 280$

N16-17/E14-17, Zone 3B. Early to Middle Austin phase, Late Prehistoric period.

Tx-2761. 41JK66/20

$1260 \pm 130$

N12-13/E14-17, Zone 3B. Early to Middle Austin phase, Late Prehistoric period.

Tx-2762. 41JK66/21

$2260 \pm 270$

N16-19/E14-17, Zone 4. Probable I ate Archaic period.

Tx-2763. 41JK66/24

$3280 \pm 360$

N12-15/E14-17, Zone 4. Probable Iate Archaic period.

Tx-2764. 41JK66/25

$3860 \pm 370$

N12-15/E14-17, Zone 3C. Transitional Archaic to early Late Prehistoric period.

Tx-2766. 41JK66/28

$730 \pm 80$

N14-15/E14-16, Zone 3B. Early to Middle Austin phase, Late Prehistoric period.

Tx-2767. 41JK66/29

$1100 \pm 140$

N14-15/E8, Zone 3. Austin phase, Late Prehistoric period.

Tx-2768. 41JK66/30

$1290 \pm 170$

N18-19/E14-15, Zone 3. Austin phase, Late Prehistoric period.

General Comment (E M Davis): dates not consistent with stratigraphy; with other evidence from site, dates suggest widespread and fairly random disturbance of deposits by rodents and people (Texas Archeol Survey Staff, 1981, p 53).

\section{Oslovsky Road site series}

Burned bone from Oslovsky Road site, 41 JK74, N side Mustang Creek, $2.4 \mathrm{~km} \mathrm{SE}$ of Ganado, in Palmetto Bend Reservoir basin $\left(29^{\circ} 2^{\prime} \mathrm{N}, 96^{\circ} 29^{\prime}\right.$ W). Residue from screening.

Tx-2769. 41JK74/1

$3950 \pm 230$

Levels 1 and 2 (0 to $40 \mathrm{~cm}$ below surface). 
Tx-2770. 41JK74/2

Levels 3 and $4(40$ to $80 \mathrm{~cm}$ below surface).

General Comment (EMD): widely separated dates indicate $0.5 \mathrm{~m}$ of cultural debris represents at least two culturally distinct Archaic assemblages (Texas Archeol Survey Staff, 1981, p 45).

\section{Lake Fork Reservoir}

Woody charcoal from sites in Lake Fork Reservoir, at confluence of Caney and Lake Fork Creeks, Wood Co, NE Texas (Bruseth \& Perttula, 1981). Coll 1978 by TK Perttula and J Bruseth and subm 1978 by J Bruseth, Archaeol Research Prog, Southern Methodist Univ, Dallas, Texas.

\section{Hines site series}

Samples from Hines site, X41WD87, in uplands $1.6 \mathrm{~km}$ from confluence of Burke and Caney Creeks, $16 \mathrm{~km}$ NE of Alba, Lake Fork Reservoir basin $\left(32^{\circ} 54^{\prime} 55^{\prime \prime}\right.$ N, $\left.95^{\circ} 38^{\prime} 56^{\prime \prime} \mathrm{W}\right)$. Pecan Grove cultural phase (Sanders focus, carly Caddoan).

\section{Tx-3043. X41WD87, \# 1}

$910 \pm 50$

Burned post in house pattern, Feature 1 .

Tx-3044. X41WD87, \#2

$410 \pm 60$

Burned timber, Feature 1, plow zone.

General Comment (JEB): Tx-3043 consistent with artifactual data from single-component Sanders focus structure. Tx-3044 should agree with -3043; sample probably out of context because of loc in plow zone.

\section{Taddlock site series}

Samples from Midden A, Taddlock site, X41WD39, on upland remnant $400 \mathrm{~m} \mathrm{~W}$ of Lake Fork Creek, $9.7 \mathrm{~km}$ NNE of Alba, Lake Fork Reservoir basin (32 $\left.51^{\prime} 46^{\prime \prime} \mathrm{N}, 95^{\circ} 36^{\prime} 42^{\prime \prime} \mathrm{W}\right)$. Pecan Grove cultural phase (Sanders focus, early Caddoan).

\section{Tx-3046. X41WD39, \#4}

$\mathbf{9 5 0} \pm \mathbf{5 0}$

Unit 25, Level 4, 30 to $40 \mathrm{~cm}$ below surface.

Tx-3047. X41WD39, \#5

$970 \pm 40$

Unit 25 , Level 5,40 to $50 \mathrm{~cm}$ below surface.

Tx-3048. X41WD39, \#6

$1010 \pm 60$

Unit 31 , Level 3, 20 to $30 \mathrm{~cm}$ below surface.

Tx-3050. X41WD39, \#8

$490 \pm 60$

Unit 18, Level 5,40 to $50 \mathrm{~cm}$ below surface.

General Comment (JEB): Tx-3046 through -3048 appropriate for singlecomponent Sanders focus midden. Tx-3050 too young; reason unknown. 


\section{Spoonbill site series}

Hickory nut shells (Carya sp) from Spoonbill site, 41 WD109, $15.5 \mathrm{~km} \mathrm{~N}$ of Quitman, on terrace of Caney Creek, in Lake Fork Reservoir basin ( $32^{\circ}$ $55^{\prime} 44^{\prime \prime} \mathrm{N}, 95^{\circ} 28^{\prime} 03^{\prime \prime} \mathrm{W}$ ). Pecan Grove cultural phase (early Caddoan).

Tx-3570. 41WD109, \#1

$1010 \pm 80$

Featurc 1, hearth.

Tx-3571. 41WD109, \#2

$950 \pm 50$

Feature 2, hearth, Level 1.

Tx-3572. 41WD109, \#3

$720 \pm 80$

Feature 2, hearth, Level 2.

Tx-3573. 41WD109, \#4

$690 \pm 70$

Feature 5, pit.

Tx-3574. 41WD109, \#5

$690 \pm 60$

Feature 9, possible smudge pit.

\section{Tx-3045. Killebrew \#3}

$1760 \pm 50$

From hearth (Feature 3), Killebrew site, X41WD64/69, $11.3 \mathrm{~km} \mathrm{NE}$ of Alba, $1.6 \mathrm{~km}$ upstream of confluence of Caney and Lake Fork Creeks, in Lake Fork Reservoir basin (32 $\left.50^{\prime} 47^{\prime \prime} \mathrm{N}, 95^{\circ} 32^{\prime} 08^{\prime \prime} \mathrm{W}\right)$. Forest Hill cultural phase (Titus focus, late Caddoan). Comment ( JEB): date $>1000 \mathrm{yr}$ too old for Forest Hill; may represent earlier Archaic occupation also evident at site (Bruseth \& Perttula, 1981, p 31).

Tx-3049. Osborn \#7

$1190 \pm 50$

Sample from Osborn site, X41WD16, on upland remnant $9.7 \mathrm{~km} \mathrm{E} \mathrm{of}$ Alba, $40 \mathrm{~m}$ W of Lake Fork Creek, in Lake Fork Reservoir basin (32 $48^{\prime} 34^{\prime \prime}$ N, $95^{\circ} 33^{\prime} 01^{\prime \prime}$ W). Midden A, Unit 10, Feature 2, 37 to $44 \mathrm{~cm}$ below surface. Lone Oak cultural phase (early Caddoan). Comment (JEB): agrees well with current consensus of earliest Caddoan.

\section{Idaho}

Tx-3698. Spalding 79/1

$2160 \pm 130$

Wood charcoal from Spalding site (10NP108), left bank Lapwai Creek $200 \mathrm{~m}$ from mouth, Nez Perce Co $\left(46^{\circ} 26^{\prime} 10^{\prime \prime} \mathrm{N}, 116^{\circ} 49^{\prime} 20^{\prime \prime} \mathrm{W}\right)$. Composite of several samples taken from floor of pit house \# 1 ; small side-notched points assoc. Coll 1979 and subm 1979 by D H Chance, Lab Anthropol, Univ Idaho, Moscow, Idaho. Comment (DHC): other dates from House 1: UGa-3308, $1530 \pm 190$; UGa-3309, $1265 \pm 70$ (Chance, pers commun). All 3 dates appear unrealistically early, as side-notched points first appear in this area ca 750 BP. Reason for discrepancy is unknown. 
Washington

Charcoal, except where otherwise noted, from sites on Lake Roosevelt, on Columbia R, NE Washington. Coll 1972 and 1978; subm 1979 by 1) H Chance. All refs to GaK and WSU dates are from Chance (pers commun).

\section{FE45 series}

Samples from 45FE45, W side Lake Roosevelt just above Kettle Falls $\left(48^{\circ} 38^{\prime} \mathrm{N}, 118^{\circ} 07^{\prime} \mathrm{W}\right)$.

\section{Tx-3494. LR72/28}

$1630 \pm 80$

Strata $2 \mathrm{~A}$ and 3 mixed, Level 4, 30 to $40 \mathrm{~cm}$; assoc with Sinaikst period materials. Comment (IHC): ca 500 yr older than expected.

\section{Tx-3495. LR72/30}

$3910 \pm 80$

Stratum 5, Level 12,110 to $120 \mathrm{~cm}$; assoc with terminal climax occupation of Ksunku period. Strat context limited vertically and very clear. Comment (DHC): other dates from Stratum 5: WSU-1420, 2960 \pm 60 ; GaK6420, 3850 \pm 140 ; on related material: GaK-7711, 3630 \pm 390 . Expected age: 360()$-3800 \mathrm{BP}$.

\section{Tx-3496. LR78/109}

$2890 \pm 340$

Area A, upper Stratum 4, Level 20; assoc with end of period of minimal occupation following Ksunku period. Comment (DHC): should be earlier than WSU-1658, $1740 \pm 70$.

Tx-3497. LR78/126

$$
1000 \pm 80
$$

Arca A, Stratum 3, Level 26-27; outer lag horizon (prehistoric fluvial redeposition). Comment (DHC): other date, GaK-7710, 750 \pm 90 , seems too late.

\section{ST65 series}

Charcoal from Site $45 \mathrm{ST} 65$ at China Bend on upper Columbia R, $40 \mathrm{~km}$ above Kettle Falls $\left(48^{\circ} 49^{\prime} \mathrm{N}, 117^{\circ} 56^{\prime} \mathrm{W}\right)$. Coll 1978 and subm 1979 by $\mathrm{D} H$ Chance.

Tx-3498. LR78/132

$6510 \pm 100$

From floor of sealed house, Area A, Stratum 7. Comment (DHC): GaK$7707,4780 \pm 140$ from same occupation.

Tx-3499. LR78/133

$$
600 \pm 70
$$

From fill of Feature 9, Area B, Stratum 4, Level 1, Chekwo component, probably of Sinaikst period. Comment (I)HC): estimated age: $1000 \mathrm{BP}$; charcoal may be later than assoc cultural material.

\section{ST94 series}

Samples from midden at 45ST94, salmon fishing site next to Kettle Falls on Columbia R $\left(48^{\circ} 38^{\prime} \mathrm{N}, 118^{\circ} 07^{\prime} \mathrm{W}\right)$. Coll 1974 and 1978 ; subm 1979 by $\mathrm{D} \mathrm{H}$ Chance. 
Tx-3500. LR74/36

$460 \pm 50$

Charcoal from Area A, base of Stratum 2, Level 3; early Shwayip period. Comment (DHC): WSU-1502, 550 \pm 70 from Stratum 3 just below.

Tx-3501. LR78/26, 78/27

$3850 \pm 100$

Sand and silt with carbon particles from Area A, base of Stratum 5, within top of fluvial cobble zone, Takumakst period. Comment (DHC): WSU-1530, 1360 \pm 100 , from level 10 just above Stratum 5 , seemed much too late; WSU-1653, $2910 \pm 160$, seems more accurate.

Tx-3502. LR74/51

$780 \pm 70$

Charcoal from Area B, Stratum 2, Level 2, above Feature 2; assoc with early Shwayip period materials.

Tx-3503. LR76/21

$1620 \pm 270$

Charcoal from Area E, Stratum 3, Level 7, Takumakst period. Comment (DHC): WSU-1 657, 1470 \pm 120 , from intrusive hearth, possibly from same level.

Missouri

Tx-2872. Crane 14-5

$920 \pm 50$

Charcoal from Level 5 (40) to $50 \mathrm{~cm}$ below surface) in Pit 14, Crane site (23SN615), 0.8km S of Crane, on Crane Creek, Stone Co $\left(36^{\circ} 53^{\prime} \mathrm{N}, 93^{\circ} 33^{\prime}\right.$ $\mathrm{W})$. From shallow pit in disturbed deposit. Coll 1977 by C Helm and M Caldwell and subm 1977 by R E Cooley, Center Archaeol Research, Southwest Missouri State Univ, Springfield, Missouri. Comment (B L Turner \& C Helm): no certain artifact assoc with pit; Woodland and Mississippian projectile points and grit-tempered pottery in adjacent deposit. Date is compatible with these finds.

\section{Tx-3016. Morgan Spring \#3}

$1390 \pm 220$

Charcoal from base of midden deposit, Morgan Spring site (230R49), W bank Elevenpoint R, ca $1.6 \mathrm{~km}$ above Hwy 142, Mark Twain Natl Forest $\left(36^{\circ} 34^{\prime} \mathrm{N}, 91^{\circ} 11^{\prime} \mathrm{W}\right)$. Outside Feature 1, base of Level 2, at N348.68 E306.11, $19 \mathrm{~cm}$ below surface; assoc with thick pottery tempered with limestone and shell. Coll and subm 1978 by C R Price, Center Archaeol Research, Southwest Missouri State Univ.

\section{Fourche Creek series}

Samples from Test Pit 1, Feature 1, Level 2, Site 23RI192, N fork Fourche Creek, $13 \mathrm{~km} \mathrm{SW}$ of Doniphan, Ripley Co $\left(36^{\circ} 34^{\prime} \mathrm{N}, 90^{\circ} 57^{\prime} \mathrm{W}\right)$. Assoc with Early Mississippian pottery (Coles Creek period, Scatters/Beckwith phase). Coll and subm 1979 by J Zarins, Center Archaeol Research, Southwest Missouri State Univ.

Tx-3608. 23RI192 \# 1

$1300 \pm 70$

Carbonized nut and wood fragments. 
Tx-3609. 23RI192 \#2

Carbon-stained earth.

Kentucky

\section{Southwest Jefferson Project series}

Samples from sites in suburbs of Louisville, S Jefferson Co. Coll 1977 by B Driskell and M B Collins and subm 1978 by M B Collins, Dept Anthropol, Univ Kentucky, Lexington.

\section{Tx-2950. 15JF18, C-5}

$$
\mathbf{1 7 0} \pm \mathbf{5 0}
$$

Charcoal, Site $15 \mathrm{JF} 18$, E bank Ohio R at river mile $6221 / 2\left(38^{\circ} 06^{\prime} \mathrm{N}\right.$, $\left.85^{\circ} 54^{\prime} \mathrm{W}\right)$. Feature $92,50 \mathrm{~cm}$ below surface. Burned clay pit, no artifacts assoc; suspected to be historic, AD 1790-1850.

Tx-3010. 15JF110, CS-5/6

$2390 \pm 70$

Carbonized wood from Site 15JF 1 10, ca $50 \mathrm{~m} \mathrm{E}$ of Ohio R, $300 \mathrm{~m} \mathrm{~N}$ of mouth of Mill Creek ( $\left.38^{\circ} 04^{\prime} 00^{\prime \prime} \mathrm{N}, 85^{\circ} 54^{\prime} 30^{\prime \prime} \mathrm{W}\right)$. Feature 21, Levels 5-9, N592.1 E703.0. Late Archaic-Early Woodland.

Tx-3013. 15JF14, C-1

$3090 \pm 150$

Carbonized wood from Site $15 \mathrm{JF} 14, \mathrm{E}$ bank Ohio $\mathrm{R}$ at river mile 621.5 , S of Louisville $\left(36^{\circ} 6^{\prime} 45^{\prime \prime} \mathrm{N}, 85^{\circ} 54^{\prime} 0^{\prime \prime} \mathrm{W}\right)$. Feature 2, Zone II, Levels 5 and 6, N769.3 E354.0. Possible Late Archaic context.

\section{JF243 site series}

Samples from Site 15JF243, E bank Ohio R at river mile $725\left(38^{\circ} 4^{\prime} 0^{\prime \prime}\right.$ $\left.\mathrm{N}, 85^{\circ} 54^{\prime} 20^{\prime \prime} \mathrm{W}\right)$. This is part of SW Jefferson Project, above.

Tx-2951. 15JF243, CN-9

$8420 \pm 110$

Charcoal from Feature 19, Zone III, ca $50 \mathrm{~cm}$ below present surface. N498 E494. Early Archaic, bifurcated-base points assoc.

Tx-3011. 15JF243, CS-17

$8440 \pm 380$

Carbonized wood from small charcoal-lined pit, Feature 153, Zone VII, N398 E506. Early Archaic, Kirk-like projectile points.

Tx-3012. 15JF243, CS-36

$\mathbf{9 4 9 0} \pm \mathbf{2 3 0}$

Carbonized wood from Feature 194, Zone XIII, N401.32 E507.8. Early Archaic, stratigraphically below Tx-3011 (above); one projectile point (Kirk variant?) assoc.

Florida

Tx-2636. Little Salt Spring 025

$12,030 \pm 200$

Wood from Little Salt Spring site (8-SO-18), ca $4 \mathrm{~km} \mathrm{NNE} \mathrm{of} \mathrm{center} \mathrm{of}$ North Port $\left(27^{\circ} 04^{\prime} 27^{\prime \prime} \mathrm{N}, 82^{\circ} 14^{\prime} 01^{\prime \prime} \mathrm{W}\right)$. From stake assoc with extinct variety of giant land tortoise; excavation $\mathrm{B} 75$, Test 1 , ca $22 \mathrm{~m}$ below msl. 
Coll 1975 and subm 1977 by C.J Clausen, General Development Foundation, Inc, North Port. Commenl (CJC): assoc tortoise shell date: Tx-2335, $13,450 \pm 190(\mathrm{R}, 1977$, v 19, p 315-316).

Tx-2637. Jones site 030

$$
810 \pm 70
$$

Bone from Glades period midden, Jones site (8-SO-85), $3.4 \mathrm{~km} \mathrm{NW}$ of Port Charlotte $\left(27^{\circ}\left(02^{\prime} \mathrm{N}, 82^{\circ} 06^{\prime} \mathrm{W}\right)\right.$. Test 1 , Level 2,10 to $20 \mathrm{~cm}$ below surface. Coll 1977 and subm 1977 by C. J Clausen.

\section{Mexico}

All samples subm 1975 by A García Cook, Monumentos Prehispánicos I N A H, Córdoba no. 45, Mexico I) F, except where noted.

\section{Tlaxcala}

\section{Los Teteles de Ocotitla series}

Charcoal from Los Teteles de Ocotitla site (T-358), on N slope La Caldera arroyo, in valley of Sierra de la Caldera, $14 \mathrm{~km} \mathrm{~N}$ of Huamantla $\left(19^{\circ} 27^{\prime}\right.$ $30^{\prime \prime} \mathrm{N}, 90^{\circ} 53^{\prime}\left(00^{\prime \prime} \mathrm{W}\right)$. Coll 1974 by A Carcía Cook. Except where noted, assocs are with materials of carly Tenanyecac phase (Teotihuacán II).

Tx-2137. T-358, 1

$2230 \pm 60$

Pit 1, E 1.2, $0.85 \mathrm{~m}$ depth, next to Burial 2.

Tx-2138. T-358, 2

$$
1780 \pm 50
$$

Pit 1, Tomb 1, Burial 4, 1.60m depth. Very early Tenanyecac (Teotihuacán IA).

Tx-2139. T-358, 3

$1840 \pm 60$

Pit 1, Tomb 1, Burial $6,1.60 \mathrm{~m}$ depth.

Tx-2140. T-358, 4

$1540 \pm 60$

Pit 1, Exterior Tomb (E side), $1.50 \mathrm{~m}$ depth.

Tx-2141. T-358, 5

$1820 \pm 60$

Pit 1, Tomb 1, upper part, $0.53 \mathrm{~m}$ depth.

Tx-2142. T-358, 6

$1690 \pm 130$

Pit 1, Tomb 1, middle part, $0.95 \mathrm{~m}$ depth.

Tx-2143. T-358, 7

$1940 \pm 60$

Pit 1, Tomb 1, middle part, $1.07 \mathrm{~m}$ depth.

Tx-2239. T-358, 43

$1810 \pm 60$

Pit 4, Level J, 2.70m depth. Transition between local Tezoquipan and Tenanyecac phases (Protoclassic). 
Tx-2240. T-358, 44

$1580 \pm 40$

Cut W, Sec C, Level D, $0.90 \mathrm{~m}$ depth. Fireplace. Beginning of early Tenanyecac phase.

Tx-2241. T-358, 45

$1990 \pm 50$

Cut W, Sec C, Level E, $1.10 \mathrm{~m}$ depth. Fireplace. Beginning of early Tenanyecac phase.

Tx-2242. T-358, 46

$1560 \pm 50$

Pit 4, Level E, $1.90 \mathrm{~m}$ depth.

Tx-2243. T-358, 47

Pit 4, Lcvel H, 2.55m depth.

$1860 \pm 40$

\section{San José Tetel series}

Charcoal from San José Tetel site (T-24), on slope $2 \mathrm{~km} \mathrm{~N}$ of Apizaco $\left(19^{\circ} 26^{\prime} 10^{\prime \prime} \mathrm{N}, 98^{\circ} 09^{\prime} 30^{\prime \prime} \mathrm{W}\right)$. Coll 1975 by A García Cook.

Tx-2245. San José Tetel 49

$1870 \pm 100$

Pit 2, Level B, $0.85 \mathrm{~m}$ depth. Early Tezoquipan phase.

Tx-2246. San José Tetel 50

Pit 2, Level C, $1.29 \mathrm{~m}$ depth. Texoloc phase.

$2470 \pm 80$

Tx-2247. San José Tetel 51

$2530 \pm 100$ phase.

Pit 2, Level I), 1.50m depth. Early Texoloc phase and/or late Tlatempa

Tx-2248. San José Tetel 52

$2230 \pm 80$

Pit 1, Level D, 0.9m depth. Late Texoloc phase.

Tx-2249. San José Tetel 53

$2130 \pm 140$

Pit 1, Level F, 1.70m depth. Texoloc phase.

San Rafael Tenanyecac series

Charcoal from San Rafael Tenanyecac site (T-288), between Nativitas Hwy to San Martin and left bank of Atoyac R, in valley at foot of Cerro Nativitas (Xochitecatl) (19 $\left.14^{\prime} 00^{\prime \prime} \mathrm{N}, 98^{\circ} 21^{\prime} 15^{\prime \prime} \mathrm{W}\right)$. Coll 1973 by R Abascal and A García Cook.

Tx-2154. San Rafael Tenanyecac 18

$2530 \pm 110$

Pit 2, Level V, 0.85m, depth. Initial Tezoquipan phase.

Tx-2155. San Rafael Tenanyecac 19

$2760 \pm 130$

Pit 2, Level IX, $1.80 \mathrm{~m}$ depth. Early Texoloc phase.

\section{Cerro Gordo de Ixtacuixtla series}

Charcoal from Cerro Gordo de Ixtacuixtla site (T-182), $1 \mathrm{~km} \mathrm{~S}$ of San Felipe Ixtlacuixtla at side of San Martin-Tlaxcala hwy $\left(19^{\circ} 19^{\prime} 19^{\prime \prime} \mathrm{N}, 98^{\circ} 22^{\prime}\right.$ 40" W). Coll 1973 by R Abascal. 
Tx-2158. T-182, 22

Pit 2, Level IV, $0.45 \mathrm{~m}$ depth. Middle Texoloc phase.

Tx-2159. T-182, 23

$2260 \pm 200$

Pit 2, Level V, $0.87 \mathrm{~m}$ depth. Early Texoloc phase.

Tx-2160. Piedra del Padre 25

$1830 \pm 60$

Charcoal from Structure 1, Level III, 0.75m depth, Piedra del Padre site (T-436), $7 \mathrm{~km}$ ESE of Terrenate $\left(19^{\circ} 29^{\prime} 15^{\prime \prime} \mathrm{N}, 97^{\circ} 51^{\prime} 10^{\prime \prime} \mathrm{W}\right)$. Early Tenanyecac phase. Coll 1974 by A García Cook.

Tx-2161. San Jorge Tezoquipan 24

$2600 \pm 1340$

Charcoal from Pit 1, Level III, 0.47m depth, San Jorge Tezoquipan site (T-263), $1 \mathrm{~km} \mathrm{~S}$ of San Jorge Tezoquipan $\left(19^{\circ} 18^{\prime} 45^{\prime \prime} \mathrm{N}, 98^{\circ} 18^{\prime} 15^{\prime \prime} \mathrm{W}\right)$. Texoloc phase. Coll 1973 by R Abascal.

Tx-2162. Tetepetla Contla 26

$2740 \pm 520$

Charcoal from strat cut, depth $3.50 \mathrm{~m}$, Tetepetla Contla site (T-336), N side of San Bernadino Contla (19 $\left.20^{\prime} 05^{\prime \prime} \mathrm{N}, 98^{\circ} 09^{\prime} 10^{\prime \prime} \mathrm{W}\right)$. Tezoquipan phase. Coll 1973 by A García Cook.

Tx-2163. Tlacatecpan Contla 27

$2480 \pm 70$

Charcoal from side of pit in Edificio 1, $3.20 \mathrm{~m}$ below surface, Tlacatecpan Contla site (T-337), $1 \mathrm{~km} \mathrm{NF}$ of San Bernadino Contla $\left(19^{\circ} 20^{\prime} 00^{\prime \prime} \mathrm{N}\right.$, $\left.98^{\circ} 08^{\prime} 47^{\prime \prime} \mathrm{W}\right)$. Transition between Tezoquipan and Tenanyecac phases. Coll 1973 by A García Cook.

Tx-2166. Las Calaveras Tlacocalpan 30

$2460 \pm 80$

Charcoal from Pit 1, Level III, 0.42m depth, Las Calaveras Tlacocalpan site (T-285), $7 \mathrm{~km} \mathrm{NE}$ of city of Tlaxcala $\left(19^{\circ} 22^{\prime} 08^{\prime \prime} \mathrm{N}, 98^{\circ} 12^{\prime} 20^{\prime \prime} \mathrm{W}\right)$. Local manifestation of Texcalac phase. Coll 1973 by A García Cook.

Tx-2250. Los Cerritos de Guadalupe 54

$1690 \pm 70$

Charcoal from Pit 2, Level C, (0.54m depth, Los Cerritos de Guadalupe site (T-85), on property of Ejido Cuamanzingo, ca $14 \mathrm{~km} \mathrm{NW}$ of Apizaco $\left(19^{\circ} 31^{\prime} 20^{\prime \prime} \mathrm{N}, 98^{\circ} 14^{\prime} 40^{\prime \prime} \mathrm{W}\right)$; alt $2535 \mathrm{~m}$. Teotihuacán phase (Classic). Coll 1975 by A García Cook.

Puebla

Tx-2165. Piedra Parada 29

$880 \pm 70$

Charcoal, probably from Level II, 1.03m depth, Piedra Parada site (P187), in pine forest $11 \mathrm{~km} \mathrm{~N}$ of Santa Rita Tlahuapan $\left(19^{\circ} 25^{\prime} 25^{\prime \prime} \mathrm{N}, 98^{\circ} 33^{\prime}\right.$ $55^{\prime \prime}$ W). Probably Tlaxcala phase. Coll 1973 by R Abascal.

\section{La Pedrera de Tlalancaleca series}

Charcoal from La Pedrera de Tlalancaleca site (P-119), $11 \mathrm{~km} \mathrm{WNW} \mathrm{of}$ San Martin Texmelucan, Puebla, above right edge of present Mexico- 
Puebla hwy $\left(19^{\circ} 19^{\prime} 10^{\prime \prime} \mathrm{N}, 98^{\circ} 32^{\prime} 52^{\prime \prime} \mathrm{W}\right)$. Coll 1973 by R Abascal and A García Cook.

Tx-2144. La Pedrera 8

$1700 \pm 80$

Pit 6, Level IV, 0.90m depth. Early Tenanyecac phase (Protoclassic).

Tx-2145. La Pedrera 9

$2840 \pm 270$

Pit 6, Level VI, $1.30 \mathrm{~m}$ depth. Late Texoloc/Early Tezoquipan phase (Late Preclassic).

Tx-2146. La Pedrera 10

$2550 \pm 1020$ sic).

Pit 6, Level IX, $2.10 \mathrm{~m}$ depth. Early Texoloc phase (Middle Preclas-

Tx-2147. La Pedrera 11

$2170 \pm 140$

Pit 9, Level IIa, $0.45 \mathrm{~m}$ depth. Early Texoloc phase.

Tx-2148. La Pedrera 12

$2330 \pm 100$

Pit 17, Level II, 0.35m depth. Early Texoloc phase.

Tx-2149. La Pedrera 13

$2280 \pm 80$ sic).

Pit 17, Level III, 0.77m depth. Late Tlatempa phase (Middle Preclas-

Tx-2150. La Pedrera 14

$2250 \pm 100$

Pit 18, Level III, $0.87 \mathrm{~m}$ depth. Early Texoloc phase.

\section{El Ameyal de la Pedrera de Tlalancaleca series}

Charcoal from El Ameyal de la Pedrera de Tlalancaleca site (P-119), $11 \mathrm{~km}$ WNW of San Martín Texmelucan, above right edge of MexicoPuebla hwy in territory of San Matías Tlalancaleca $\left(19^{\circ} 18^{\prime} 30^{\prime \prime} \mathrm{N}, 98^{\circ} 31^{\prime}\right.$ 09" W). Coll 1973 by A García Cook. All samples from "Elemento 7."

Tx-2151. P-119, 15

$1820 \pm 90$

Sq N5/E3, Level IV, $1.17 \mathrm{~m}$ depth.

Tx-2152. P-119, 16

$1860 \pm 100$

Sq N4/E3, Level III, 1.13m depth.

Tx-2153. P-119, 17

$2200 \pm 170$

Sq N2/E7, Level V, $1.77 \mathrm{~m}$ depth.

Tx-2244. P-119, 48

$1670 \pm 80$ sic).

Sq N6/E3, Level III(?), $1.15 \mathrm{~m}$ depth. Tezoquipan phase (Protoclas-

\section{Gualupita las Dalias series}

Charcoal from Gualupita las Dalias site (P-164), on tableland surrounded by ravines, $10 \mathrm{~km}$ NE of Santa Rita Tlahuapan $\left(19^{\circ} 25^{\prime} 20^{\prime \prime} \mathrm{N}, 98^{\circ}\right.$ $\left.31^{\prime} 20^{\prime \prime} \mathrm{W}\right)$, alt $2650 \mathrm{~m}$. Coll 1973, 1975 by A García Cook. 
Tx-2164. Gualupita las Dalias 28

$2180 \pm 60$

From tunnel in interior of pyramidal structure, $4.0 \mathrm{~m}$ depth. Tezoquipan phase (Preclassic).

Tx-2251. Gualupita las Dalias 55

$2310 \pm 90$

Pit A, Level B, 0.37m depth. Late Tezoquipan phase (Protoclassic).

Tx-2252. Gualupita las Dalias 56

$2280 \pm 100$

Pit A, Level I), $0.90 \mathrm{~m}$ depth. Tezoquipan phase.

Tx-2253. Gualupita las Dalias $\mathbf{5 7}$

$2350 \pm 100$ phases.

Pit 3, Level H, $2.07 \mathrm{~m}$ depth. Farly Tezoquipan and late Texoloc

Tx-2254. Gualupita las Dalias 58

$1960 \pm 70$

Pit 4, Level F, 2.05m depth. Early Tezoquipan phase.

Tx-2255. Gualupita las Dalias 59

$1960 \pm 70$

Pit 6, Level G, 1.80m depth. Early Tezoquipan phase.

Tx-2256. Gualupita las Dalias 60

$2150 \pm 80$

Pit 4, "Elemento 1," 2m depth. Early Tezoquipan phase.

Tx-2257. Gualupita las Dalias 61

$1970 \pm 80$

Pit 5, "Elemento 1," 2.25m depth. Early Tezoquipan phase.

Tx-2258. Gualupita las Dalias 62

$2180 \pm 80$

Pit 8, "Elemento 2," 1.42m depth. Early Tezoquipan phase.

Tx-2259. Gualupita las Dalias 63

$2360 \pm 70$

Pit 11, "Elemento 3-A," 2.07m depth. Early Tezoquipan phase.

Tx-2260. Gualupita las Dalias 64

$1980 \pm 60$

Pit 13, "Elcmento 4-A," $1.45 \mathrm{~m}$ depth. Tezoquipan phase.

Tx-2261. Gualupita las Dalias 65

$2490 \pm 80$

Pit 14, "Elemento 4-A," 1.80m depth. Early Tezoquipan phase.

\section{Matlactzingo series}

Charcoal from Matlactzingo site (P-62), at confluence of Atoyac and los Chorros Rivers $\left(18^{\circ} 49^{\prime} 40^{\prime \prime} \mathrm{N}, 98^{\circ} 00^{\prime} 30^{\prime \prime} \mathrm{W}\right)$. Final epoch of local Classic culture. Coll 1975 by A Salas Porras and P Dávila Cabrera and subm 1975 by P Dávila Cabrera.

Tx-2227. P-62, 31

$1550 \pm 60$

Pit 3, Level VI, 0.54m depth.

Tx-2228. P-62, 32

$2250 \pm 60$

Pit 4, Level XII, $2.48 \mathrm{~m}$ depth. 
Tx-2229. P-62, 33

Pit 4, Level XIII, $2.66 \mathrm{~m}$ depth. Sample was split and 2 parts prepared and run separately: $480 \pm 150,670 \pm 160$; date is average.

\section{Tepeaca Viejo series}

Charcoal from Tepcaca Viejo site (P-8), in canyon at $\mathrm{E}$ end of Tepeaca $\mathrm{mt}$ ridge, $1 \mathrm{~km} \mathrm{NW}$ of Tepeaca $\left(18^{\circ} 58^{\prime} 25^{\prime \prime} \mathrm{N}, 97^{\circ} 55^{\prime}\left(00^{\prime \prime} \mathrm{W}\right)\right.$. Assoc with ccramics (principally polychrome) of Mixteca-Puebla culture. Coll 1975 by S Mesa Dávila, A Salas Porras, and D Z de Dávila; subm 1975 by P Iávila Cabrera, Fundación Alemana Inv, Mexico City.

Tx-2230. P-8, 34

$2100 \pm 50$

Pit 3, Level IV, $0.35 \mathrm{~m}$ depth.

Tx-2231. P-8, 35

$610 \pm 60$

Pit 1, Level IV, 0.50) depth.

Tx-2232. P-8, 36

$1670 \pm 50$

Pit 2, Lcvel VIII, 1.22m depth.

\section{Los Teteles de San Miguel series}

Charcoal from Los Teteles de San Miguel site (P-6), ca 500M NE of Cuauhtinchan $\left(18^{\circ} 57^{\prime} 25^{\prime \prime} \mathrm{N}, 98^{\circ} 00^{\prime} 00^{\prime \prime} \mathrm{W}\right)$. Similar to Texoloc phase. Coll and subm 1975 by P Dávila Cabrera.

Tx-2233. P-6, 37

$2830 \pm 180$

Pit 4, Level IV, $1 \mathrm{~m}$ depth.

Tx-2234. P-6, 38

$2390 \pm 50$

Pit 4, Level IV, $1.10 \mathrm{~m}$ depth.

\section{Capulac Concepción series}

Charcoal from Capulac Concepción site (P-211), in Poblano Valley on Hacienda Las Vegas, 3km NW of Amozoc (19 $\left.03^{\prime} 40^{\prime \prime} \mathrm{N}, 98^{\circ} 02^{\prime} 40^{\prime \prime} \mathrm{W}\right)$. Local Te\%oquipan phase (Protoclassic). Coll 1975 by A García Cook.

Tx-2235. P-211, 39

$1550 \pm 90$

Pit 3, upper Level I) (in banquette), $1 \mathrm{~m}$ depth.

Tx-2236. P-211, 40

$680 \pm 110$

Pit 1, base of Level A, $0.50 \mathrm{~m}$ depth.

Tx-2237. P-211, 41

$480 \pm 60$

Pit 2, Level D) (in banquette), $1.30 \mathrm{~m}$ depth.

Tx-2238. P-211, 42

$\mathbf{7 9 0} \pm \mathbf{5 0}$

Pit 4, fireplace in structure assoc with ball game, $0.30 \mathrm{~m}$ depth. 


\section{Guatemala}

Charcoal from sites in vicinity of Santa Cruz del Quiche. Subm 1980 by K L. Brown, Dept Anthropol, Univ Houston, Houston, Texas.

\section{Gumarcaaj series}

Samples from Gumarcaaj site (47-40-191), ca $3 \mathrm{~km}$ W of Sta Cruz del Quiche $\left(15^{\circ} 01^{\prime} \mathrm{N}, 91^{\circ} 10^{\prime} \mathrm{W}\right)$. Estimated age: AD 1350-1524 (Spanish conquest). Coll 1978 by K I. Brown.

Tx-3813. Gumarcaaj 78-7219

$700 \pm 40$

Unit 9/37B, Level 19, elev 45.71 to $45.61 \mathrm{~m}$.

Tx-3814. Gumarcaaj 78-6911

Unit 25/19H, Level 2.

Tx-3815. Gumarcaaj 78-7141

Unit $31 / 19 \mathrm{H}$.

Tx-3816. Gumarcaaj 78-5888

Unit $1 / 37 \mathrm{~B}$. Sample split and 2 parts run independently: $850 \pm 50$, $750 \pm 60$; date is average.

\section{Greater Utatlan series}

Samples from sites at Greater Utatlan, ca $3 \mathrm{~km}$ W of Sta Cruz del Quiche $\left(15^{\circ} 01^{\prime} \mathrm{N}, 91^{\circ} 10^{\prime} \mathrm{W}\right)$. Estimated age: $\mathrm{AD} 1350$ to Spanish conquest. Coll 1978 by T Babcock.

Tx-3817. 47-41-290, 78-4989

$730 \pm 80$

Charcoal, Unit 6, Level 7, beneath slab structure in tomb.

Tx-3826. 47-41-290, 78-4956

$510 \pm 100$

Charcoal, Unit 6, Level 2, from ash and sherd lens overlying burials intrusive above tomb.

Tx-3818. 47-41-336, 78-4735

$1210 \pm 60$

Charcoal, Unit 7, Level 11, beneath lowest mound floor, $\mathrm{N}$ end of Pakaman site.

Tx-3820. 47-41-307, 78-2362

$1100 \pm 80$

Charcoal from burial pits E of Rescuardo. Unit 2, Level 6.

Tx-3821. 47-41-317, 78-3971

$3030 \pm 230$

Charcoal from burial pits cut through midden on N slope of Pakaman site. Unit 4, Level 5.

Tx-3822. 47-41-320, 78-3137

$1070 \pm 100$

Wood carbon from base of mound fill in copper-working area, Unit 7 , Level 9 . 
Tx-3823. 47-31-265, 78-0690

$1440 \pm 230$

Carbon from obsidian workshop on La Rochela, Unit 2, Level 5.

Tx-3824. 47-40-039, 78-0999

$2220 \pm 50$

Carbon assoc with whole vessels within feature in Unit 1, Level 4.

Tx-3825. 47-41-223, 78-2363

$1450 \pm 50$

Charcoal from burial pit of structure on Ovan Rojas, Unit 5, Level 8.

\section{Chitinamit series}

Samples from Chitinamit site (23-43-222), $3.5 \mathrm{~km} \mathrm{~N}$ of Sta Cruz del Quiche $\left(15^{\circ} 07^{\prime} \mathrm{N}, 91^{\circ} 04^{\prime} \mathrm{W}\right)$. Estimated dates: AD 700-900, time of initial conquest of Quiche area by Toltecs. Coll 1978 by L. Sutro.

Tx-3828. 78-7207

Early structure floor, Unit 1/1 M, Level 8.

$$
2340 \pm 50
$$

Tx-3829. 78-7204

$1200 \pm 60$

Basal occupation for site, Unit 2/2M, Level 22.

Tx-3830. 78-7206

Early structure floor, Unit 3/1 M, Level 4.

$2090 \pm 40$

Tx-3831. 78-3207

$1080 \pm 30$

Within retaining wall of platform, Unit $1 / 1 \mathrm{~W}$, Level 13 .

Tx-3832. 78-1407

$1340 \pm 60$

Below Feature 18, Unit 20N/16W, Level 7.

Tx-3833. 78-1382

$1150 \pm 90$

From early cut stone structure, Unit $6 / 7 \mathrm{M}$, Level 4 .

Tx-3834. 78-3226

$1360 \pm 40$

From hearth of palace floor, Unit 26N/30W, Level 12.

Tx-3835. 78-3147

$1200 \pm 50$

From hearth residence zone, Unit $7 \mathrm{~N} / 34 \mathrm{~W}$, Level 7 .

Tx-3836. 78-3149

$\mathbf{5 8 0} \pm \mathbf{5 0}$

From residence terrace, Unit $16 \mathrm{~N} / 44 \mathrm{E}$, Level 8.

Tx-3837. 78-4725

$1400 \pm 40$

From hearth residence, Unit 2N/68E, Level 12.

Tx-3838. 78-4721

$1540 \pm 40$

From residence terrace, Unit $46 \mathrm{~N} / 88 \mathrm{E}$, Level 3. 


\section{Chujuyab series}

Samples from Chujuyab site (36-14-015), pre-Toltec mound, estimated age: AD $700-800$, in Chujuyab Valley $15 \mathrm{~km} \mathrm{NE}$ of Sta Cruz del Quiche $\left(15^{\circ} 06^{\prime} \mathrm{N}, 91^{\circ} 03^{\prime} \mathrm{W}\right)$. Coll 1978 by T Majewski.

Tx-3840. 78-3196

$1520 \pm 110$

From possible pre-mound construction phase, Unit 5/IVm, Level 3.

Tx-3841. 78-4266

$1270 \pm 50$

From plaza between Mounds $3 \& 5$, Unit 70E/88S, Level 9.

Tx-3842. 78-3160

$1380 \pm 60$

Assoc with mound retaining wall, Unit 3/Vm, Level 8.

Tx-3843. 78-3193

$1380 \pm 60$

Near edge of mound, Unit $1 /$ IVm, Level 3.

Tx-3844. 78-3158.

$1320 \pm 60$

From part of mound retaining wall, Univ $11 / \mathrm{IVm}$, Level 5.

Tx-3845. 78-3155

$1650 \pm 70$

Between V-shaped rock and semi-dressed mica slab above it; Unit 4/ IIm, Level 3.

Tx-3846. 78-0108

$1560 \pm 100$

From ceramic feature, Unit $24 \mathrm{E} / 34 \mathrm{~N}$, Level 6.

Peru

\section{Huaricoto series}

Carbonized or burned plant tissue samples from Huaricoto site (PAn 3-35) in Marcará, Prov Carhuaz, Dept Ancash (9 $\left.19^{\prime} 57^{\prime \prime} \mathrm{S}, 77^{\circ} 36^{\prime} 57^{\prime \prime} \mathrm{W}\right)$. Ceremonial center, late Preceramic to beginning of Middle Horizon. Coll 1978 and subm 1979 by R I. Burger, Calif State Univ, Hayward.

Tx-3580. Huaricoto \#2

$4500 \pm 180$

Huaricoto IIA, Sec Iglesia, from ceremonial hearth, Floors D/E; midEarly Horizon.

Tx-3581. Huaricoto \#5

$4770 \pm 200$

Huaricoto III, Sec Camino, from platform surrounding probable late Preceramic ceremonial hearth; stratigraphically below early Middle Horizon structures.

Tx-3582. Huaricoto $\# \mathbf{6}$

$2540 \pm 130$

Huaricoto IVC, Sec Toril, Level 7b, 130 to $140 \mathrm{~cm}$, oustide of monumental wall, Early Horizon. 
Tx-3583. Huaricoto \#7

Huaricoto IVC, Sec Toril, Level $6 \mathrm{~b}, 120$ to $130 \mathrm{~cm}$, outside of Early Horizon monumental walls.

\section{REFERENCES}

Albert, L E, 1981, Ferndale Bog and Natural Lake: five thousand years of environmental change in southeastern Oklahoma: Oklahoma Archaeol Survey, Studies in Oklahoma's past no. 7, Univ Oklahoma, Norman.

Baker, V R, Kochel, R C, Patton, P C and Pickup, G, 1983, Paleohydrologic analysis of Holocene flood slack-water sediments, in Collinson, J D and Lewin, J, eds, Modern and ancient fluvial systems: Special Pub no. 6, Oxford, Internatl Assn Sedimentologists, p 229-239.

Baker, V R, Pickup, $G$ and Polach, H A, 1983, Desert paleofloods in central Australia: Nature, v 301, p $502-504$.

Baskin, B J, (ms) 1978, Test excavations at a prehistoric stratified campsite, Big Bend National Park, Brewster County, Texas: Rept subm to Natl Park Service, Southwest Regional Office, Santa Fe, New Mexico.

Broecker, W S and Kaufman, A, 1965, Radiocarbon chronology of Lake Lahontan and Lake Bonneville II, Great Basin: Geol Soc America Bull, v 76, p 537-566.

Bruseth, J F and Perttula, T K, 1981, Prehistoric settlement patterns at I ake Fork Reservoir: Texas Antiquities permit series, Rept no. 2; Archaeol Research Prog, Southern Methodist Univ, Dallas, and Texas Antiquities Comm, Austin.

Fox, 1) E, 1979, Archacological investigations of two prehistoric sites in the Coleto Creek drainage, Coliad County, Texas: Center Archaeol Research, Archaeol Survey rept 69, Univ Texas, San Antonio.

Gerstle, A, Kelly, T C and Assad, C, 1978, The Fort Sam Houston Project: an archaeological and historical assessment: Center Archaeol Research, Archaeol Survey rept 40, Univ Texas, San Antonio.

Katz, S, 1977, An archacological test excavation at John James Park, City of San Antonio, Texas: Center Archaeol Research, Archaeol Survey rept 38, Univ Texas, San Antonio. 1978, Test cxcavations at 41 CU97, in Katz, P R, An inventory and assessment of archaeological sites in the high country of Guadalupe Mountains National Park, Texas: Center Archaeol Research, Archaeol Survey rept 36, Univ Texas, San Antonio, p 81-91.

Texas Archaeological Survey Staff, 1981, Phase III prehistoric archeological research within Palmetto Bend Reservoir, Jackson County, Texas: Texas Archacol Survey Research Paper no. 82, Univ Texas, Austin. 